\title{
Design Of Heat-Activated Reversible Integral Attachments For Product-Embedded Disassembly
}

\author{
Ying Li, Kazuhiro Saitou, Noboru Kikuchi, Steven J. Skerlos \& Panos Y. Papalambros \\ Department of Mechanical Engineering \\ University of Michigan \\ Ann Arbor, MI 48109-2125, USA \\ \{yingli, kazu, kikuchi, skerlos, pyp\}@engin.umich.edu
}

\begin{abstract}
Disassembly is a fundamental process needed for component reuse and material recycling in all assembled products. Integral attachments, also known as "snap" fits, are favored fastening means in design for assembly (DFA) methodologies, but not necessarily a favored choice for design for disassembly. In this paper, several protorype designs of a new class of integral attachments are presented, where the snapped joints can be disengaged by the application of localized heat sources. The design problem of reversible integral attachments is posed as the optimization of compliant mechanisms actuated with localized thermal expansion of materials. The Homogenization Design Method is utilized to obtain an optimal structural topology that realizes a desired deformation of snapped featuers for joint release. The obtained optimal topologies are simplified to emhance the manufacturability for the conventional injection molding technologies. Results of the example designes are verified by finite element analyses.
\end{abstract}

\section{Introduction}

Driven by increasing social pressure to heighten enviromental consciousness, design for disassembly has become one of the most significant challenges in the modern product design process. This is especially true in the consumer electronics industry due to high production volumes and characteristically short time scales of technological obsolescence. A novel and effective way to enhance the disassemblability of products is to embed a desired disassembly process in the products when they are manufactured [1][2][3].

Integral snap-fit attachments [4][5][6][7] have been widely used as substitues for separate fasteners for the purpose of design forassembly (DFA)[8][9]. However, snap fits are not necessarily a favored choice fore design for disassembly since they are often difficult to disengage without inherent destruction of the components [10]. While some snap-fits are designed to be reverisble (eg., battery covers for cellular phones), they require the application of auxiliar forces in a direction different from the insertion deiretion in order to unlatch the snapping features.

The motivation of this research is to develop an enabling technology for product-embedded disassembly by designing a new class of reversible integral attachments (RIA) that can be detached by the application of loalized heat. The designs utilizethe thermal expansion of materials to induce the deformation of snapping features, thereby releasing the engaged joints, and require no special materials or manufacturing technologies. The design comprise of two major parts: a plastic part and a metal part. The plastic part is the main component of the snap-fit which includes a deflection mechanism (a latch) and retention mechanism (a catch) to be engaged with the counterpart. The metal part is a thermomechanial transducer that works as a thermal force applicatior (TFA). A TFA integrated with the engaging plastic part, is heated and the resulting thermal deformation induces the release of the snapped joint, through the transmision of the deformation to the deflection mechanism in the plastic part. (Figure 1 shows a simple illustration of such a design.) The Homogenization-based topology optimization technique developed for compliant mechanism design [11][12] is applied to design these two components. The results are combined and simplified to enhance manufactuability of the plastic part with the conventional injection molding technilogy. The simplified designs are then verified through finite element thermal analysis. Several examples of new designs are presented to illustrated the design concepts.

\section{Background}

\subsection{Design for Product Embedded Disassembly}

Academic investigations into the concept of "design for disassembly" began in the late 1980s, largely driven by successes with "design for assembly" earlier in the decade. Unfortunately, many guidelines suggested by the "design for assembly" methodology do not apply to "design for disassembly," for instance [10][13][14][15] : 
- Rapid attachment fasteners employed in assembly may not be suitable for disassembly. For example, glue can be used to easily join two plastic parts, but the resulting joints cannot be detached without damaging both parts.

- Products designed for assembly may not allow easy access to the specific components to be recovered. For example, a product may require the removal of 100 components to reach one component with high recycling value. In such cases, disassembly may not be economically feasible.

- The condition of products at disassembly may be different from the conditions under which the products were initially assembled. For example, wear or corrosion may make bolted joints extremely hard to detach.

- Due to the significant spatial and temporal distance between the assembly and disassembly processes, it may not be immediately obvious how to disassemble a part.

- Parts that appear similar may require completely different approaches to disassembly.

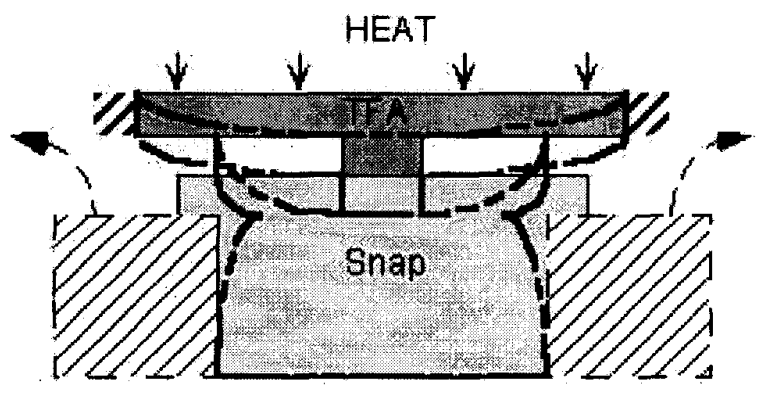

Figure 1: Design concept of a heat activated RIA.

Efforts to overcome these disassembly obstacles through the concept of "product-embedded disassembly," or "self-disassembly," have recently stated appearing in the literature. Product-embedded disassembly gives a product the ability to take itself apart. Chiodo et al [2] demonstrated the feasibility of a self-disassembly strategy for consumer electronic products using fastener screws made of a special shape memory alloy (SMA) polymer. Masui et al [1] demonstrated the self-disassembly of a CRT using nichrome wire embedded in the component glass along the desired boundary for separation. Although these examples were effective in the particular cases presented, both methods lack generality since they require the use of specialized and costly materials such as SMA polymers.

\subsection{Topology Optimization of Compliant Mechanisms}

Compliant mechanism is the type of mechanism that uses elastic deformation as the source of motion. Topology optimization is a continuum synthesis approach of design compliant mechanisms.

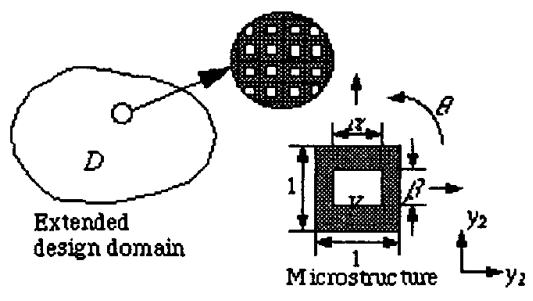

Figure 2: Homogenization Design Method [16].

The topology optimization problems is formulated as a problem of finding the optimal distribution of material properties in an extended fixed domain where some structure cost function is maximized. This technique is applied based on the homogenization method where. a microstructure proposed by Bends $\phi$ e and Kikuchi [16] is defined at each point of the domain which is a unit cell with a rectangular hole inside (Figure 2). The use of microstructure allows the intermediate materials rather than only void or full material in the final solution. The design variables are the dimensions $\alpha, \beta$ and the orientation $\theta$ of the micro-hole. In this sense the problem is to optimize the material distribution in a perforate domain with infinite microscope voids. The effective properties of the porous material, are calculated using the material homogenization methods [17].

Initially developped as a design method for stiffest structure, this Homogenization Design Method (HMD) has been extended on design of compliant mechanisms [11]. Both requirements of flexibility and stiffness are formulated into the topology optimization problem as a multi-objective function. Mutual mean compliance, which based on the reciprocal theorem in linear elasticity theory [18][19], and mean compliance are the two design objectives. In the effort of producing ECO-structure, thermal actuators made of recyclable materials [20] has also been designed. In the research a sepecially designed composite material (material with negative thermal expansion coeficient) has been used to induce a desired deformation from a global temperature change of the structure. 


\section{Design of Heat Activated Snap-Fit}

In this research, the design problem of reversible integral attachments is posed as the optimization of the compliant mechanisms actuated with localized thermal expansion of materials. For simplicity of thermal analysis, a local uniform temperature distribution is assumed near the heat source. Therefore, two materials with different thermal properties (plastic and metal) can be separately designed and integrated at a later stage. Metal material with relatively high thermal expansion coefficient and thermal conductivity has been uniformly heated to simulate localized thermal actuation, while plastic material remains unheated, i.e., deforms without any thermal stresses/strains. In this research, The following materials are chosen as design materials:

$$
\begin{aligned}
& \text { Brass : } E=106 G P a, v=0.3, \alpha=1.65 \mu /{ }^{\circ} \mathrm{C} \\
& \text { Polypropylen (PP): } E=1.6 G P a, v=0.45
\end{aligned}
$$

Both of them are assumed to be deformed within the elastic range.

\subsection{Thermal Force Applicator}

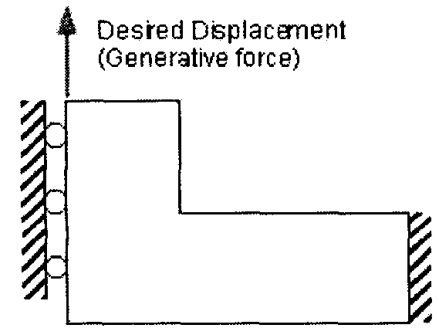

Figure 3: Design domain of TFA.

The metal part functions as a transducer of thermal energy to mechanical energy (force) via thermomechanical structural deformation. The design problem is to find optimal layout in the design domain, illustrated in Figure 3 , to achieve maximum displacement at the top in the horizontal direction when the whole material is heated uniformly. Based on the HMD for thermal actuated mechanism [20], the optimization problem is formulated as:

$$
\begin{aligned}
& \max _{\alpha, \beta, q} f=\frac{L^{\prime}\left(\mathbf{u}^{2}\right)}{L^{3}\left(\mathbf{u}^{3}\right)} \\
& \text { Subject to: } \quad L^{t}\left(\mathbf{u}^{\prime}\right)=\int_{\Gamma} \mathbf{t}^{\prime} \cdot \mathbf{u}^{\prime} d \Gamma=\int_{\Omega} \varepsilon\left(\mathbf{u}^{\prime}\right) \mathbf{E}^{H} \varepsilon\left(\mathbf{u}^{\prime}\right) d \Omega \\
& V=\int_{\Omega}(1-\alpha \beta) d \Omega \leq \bar{V} \\
& 0 \leq \alpha \leq \bar{\alpha}<1 \\
& 0 \leq \beta \leq \bar{\beta}<1
\end{aligned}
$$

where $\Omega$ represents the extended design domain in HMD. $\mathbf{u}$ and $\mathbf{t}$ are displacement vector and traction in the design domain. $\varepsilon$ is the strain field and $\mathbf{E}^{H}$ is homogenized stiffness matrix.

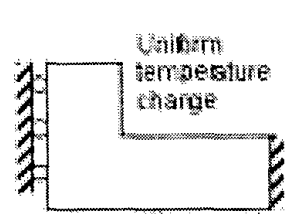

TI

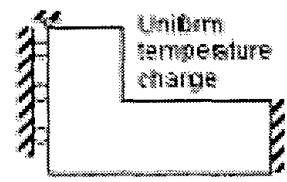

(3)

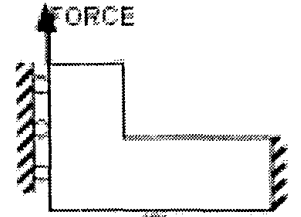

(2)
Figure 4: Loading cases considered for TFA.

$L^{i}\left(\mathbf{u}^{j}\right)$ is the energy form for mutual mean compliance and mean compliance. When $i=j$, it stands for mean compliance (stiffness measure), otherwise it stands for mutual mean compliance between two loading cases $i$ and $j$ (flexibility measure). The loading cases considered in this case is shown in Figure 4.

The first constraint satisfies the equilibrium equation, which in the topology optimization procedure is solved by finite element analysis. The rest of constraint are the volumn constraint and side constraints which is necessary in order to avoid ill-posed stiffness matrix.

Once the maximum displacement is achieved, being connected with the snapping mechanism part, TFA will be able to provide a significant force input.

Topology optimization result is shown in Figure 5, and it is simplified at a later stage and combined with plastic mechanism.

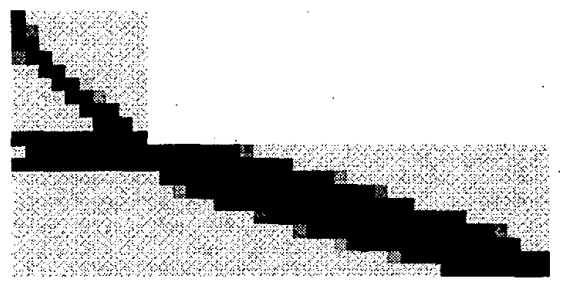

Figure 5: Optimal configure of TFA.

\subsection{Snapping mechanisms}

While the TFA may remain the same for individually different snap-fit, snapping mechanism can be designed 
differently for diversified conditions and usages. Four examples of design with different boundary condition and heat source locations are presented in the paper.

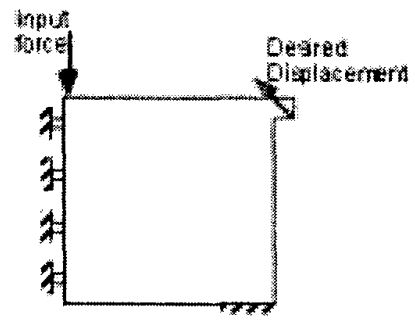

Figure 6: Design domain of Example 1.
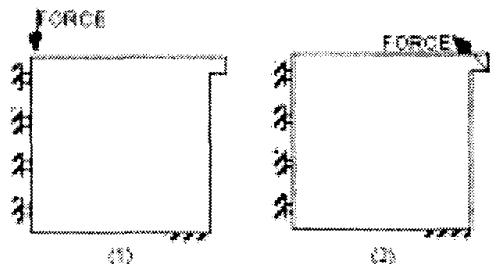

k

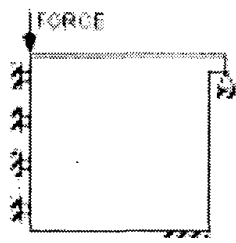

(3)

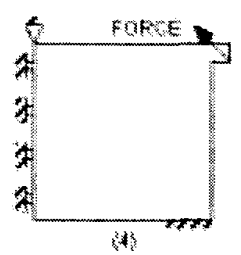

Figure 7: Loading case in Example 1.

Design domain for example 1 is shown in Figure 6. The TFA is supposed to be put on the top of the mechanisms, which is equivalent to a force to be input from center top point of the design domain. The design goal is to generate maximum displacement in the upper right direction at the hang-over portion, which means when TFA is heated and provide a force input, the mechanism can be deformed to release the snap-fit. The topology optimization problem is the same as the one in 3.1 , however with a different multiobjective function:

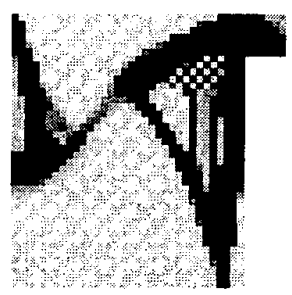

Figure 8: Optimal configuration in Example 1.

$$
\max f=\frac{L^{1}\left(\mathbf{u}^{2}\right)}{w_{s} L^{3}\left(\mathbf{u}^{3}\right)+\left(1-w_{s}\right) L^{4}\left(\mathbf{u}^{4}\right)}
$$

In the compliant mechanism design, four loading cases are considered (Figure 7) as being reflected in the multiobjective function. $w_{s}$ is the weighting factor to balance between the two stiffness requirements. Figure 8 is the optimal configuration is obtained with $w_{s}=0.6$.

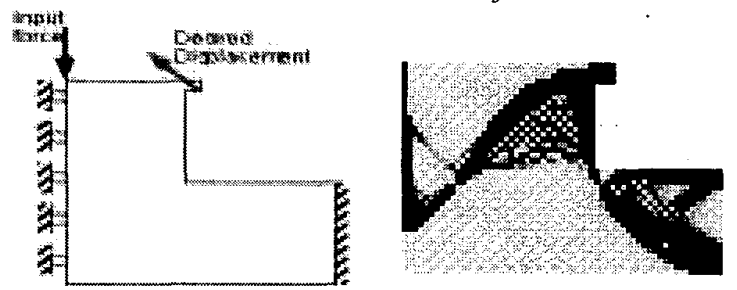

Figure 9: Design domain and optimal configuration in Example 2.
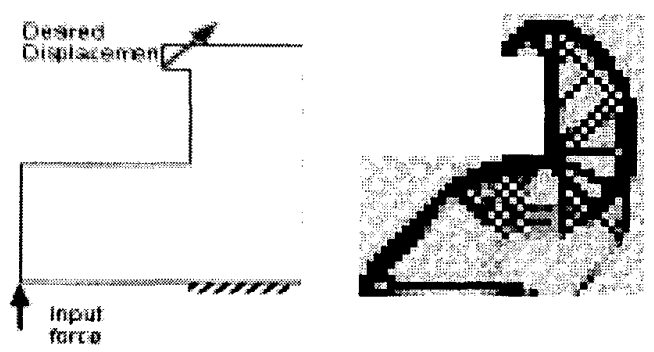

Figure 10: Design domain and optimal configuration in Example 3.

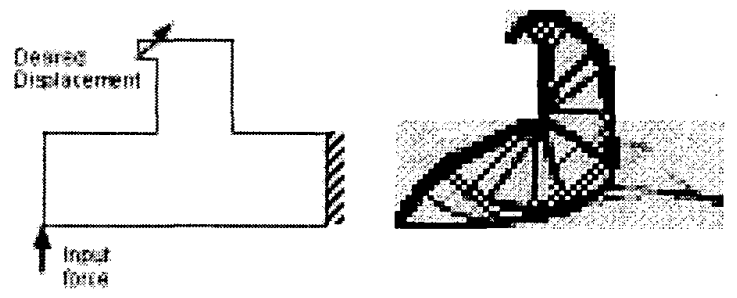

Figure 11: Design domain and optimal configuration in Example 4.

Similarly, example2 through example 4 can be formulated. Only design domains and optimal topologies are presented as in Figure 9 through 11.

\subsection{Simplified Results and Verification}

In order to enhance manufacturability, simplification scheme is applied to the optimal configurations from previous sessions. Since the major manufacturing processes considered here is the economic injection molding, the simplifications have been conducted by 
removing materials which may cause internal cavatives and re-shaping of structural members to avoid negative angles in molding. In Figure 12, simplified TFA and simplified snapping mechanisms are integrated together to illustrate final designs.
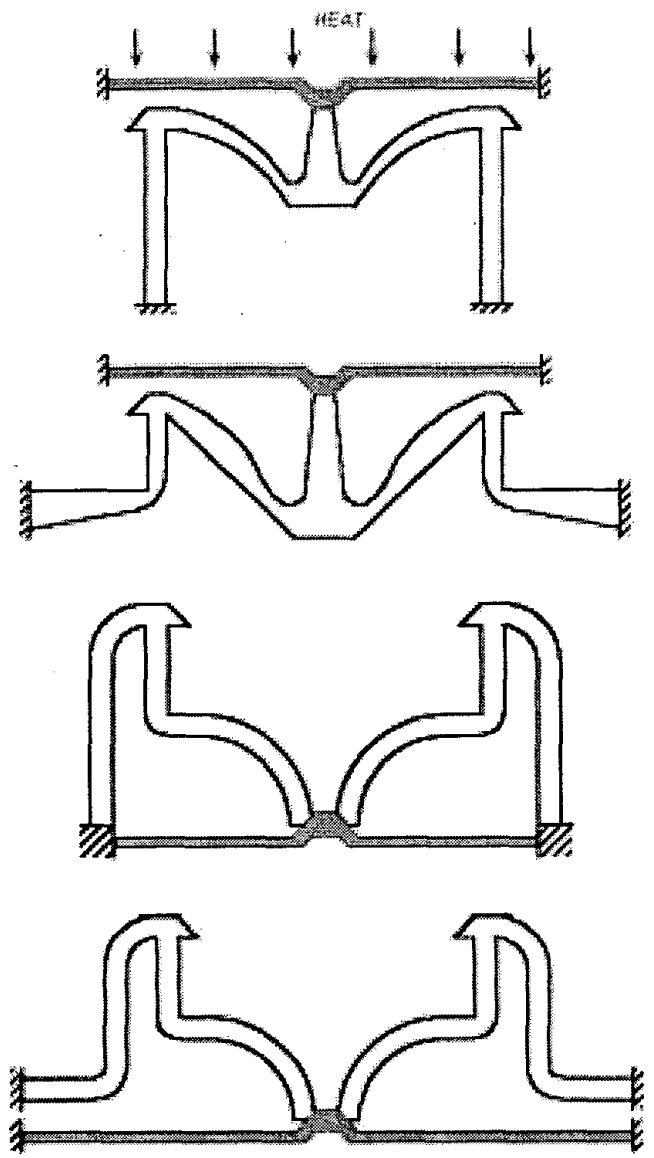

Figure 12: Final designs after simplification.

To verify that the simplified designs can still perform a desired function, static thermal analyses with a comercial FEM software ABAQUS/Standard are conducted. As shown in Figure 13, the deformed shapes verified that when the TFA part is heated, each snapping mechanism deformed in the direction of disengagement. While the same deformation can occur by directly applyging an external force without TFA, the mating parts cannot be separated since the force pushes them against each other. With TFA, the matng parts can be easily separated after deformation, since TFA exerts an input force to the plastic parts as an internal force.

\section{Discussions and Conclusions}

In this research, several prototype designs of heatactivated reversible integral attachements (RIE) have been presented. The design method is based on a topology optimization technique and a simplification scheme. The proposed design is composed of two major components made of metal and plastic materials, which are seperately designed and combined. However, the method may be further improved by taking advantage of time-transient heat transfer effects to eliminate the necessity of using two materials with different thermal conductivity. The improved design method will be able to generate even more economic and efficient RIEs for product-embedded disassembly.
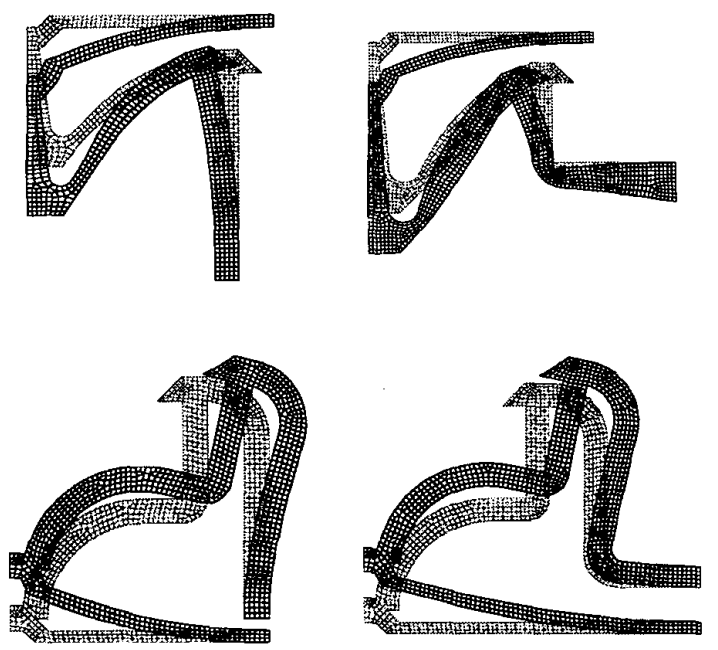

Figure 13: Verifications of final designs with ABAQUS.

In this work, it is assumed that all materials are deformed within elastic range, and stress analysis is not refered in the verification stage. A further refinement of design process can also include a post-topology shape optimization to reduce stress concentration and improve performances of the mechanism.

Examples shown in this paper are just part of the possilbe snap-fit designs. More design can be generated by applying other boundary conditions and heat locations to generate a family of snap-fits. It is expected these basic designs can be adopted in a wide range of products with minor parametric design changes, in order to realized the products with an embedded disssembly means for component resue and mterial recycling. 


\section{ACKNOWLEDGMENT}

The authors gratefully acknowledge the Institute for Environmental Sciences, Engineering and Technology (IESET) at the University of Michigan, for a partial support for this work.

\section{REFERENCES}

[1] [Masui K., Mizuhara, K. Ishii, K. and Rose, C. (1999), "Development of Products Embedded Disassembly Process Based on End-of-Life Strategies", Proceedings of the EcoDesign '99: First International Symposium on Environmentally Conscious Design and Inverse Manufacturing, pp. 570-575.

[2] Chiodo, J., Billett, E. and Harrison D. (1999), "Preliminary Investigation of Active Disassembly Using Shape Memory Polymers," Proceedings of the EcoDesign '99: First International Symposium on Environmentally Conscious Design and Inverse Manufacturing, pp. 590-596.

[3] Zhang, H., Kuo T., Lu. H, Huang, S. (1997), "Environmentally Conscious Design and Manufacturing: A State-of-the-Art Survey," Journal of Manufacturing Systems, Vol.. 18, No. 5, pp. 352-371.

[4] Turnbull, V. (1984), "Design Considerations for Cantilever Snap-Fit Latches in Thermoplastics", Proceedings of the Winter Annual Meeting of ASME, 84-WA/Mats-28, pp. 1-8.

[5] Wang, L., Gabriele, G. and Luscher, A. (1995), "Failure Analysis of a Bayonet-Finger Snap-Fit", Proceedings of the ANTEC '95, pp. 3799-3803.

[6] Larsen G. and Larson, R. (1994), "Parametric FiniteElement Analysis of U-Shaped Snap-Fits", Proceedings of the ANTEC' '94, pp. 3081-3084.

[7] Genc, S., Messler, R.W. Jr, \& Gabriele, G. A. (1998), "A Systematic Approach to Integral Snap-Fit Attachment Design", Research in Engineering Design 10: 84-93

[8] Boothroyd, G., Dewhurst, P. (1983), Design of Assembly Handbook, University of Massachusetts, Amherst, MA.

[9] Miyakawa, S., Ohashi, T. (1986), The Hitachi Assemblability Method, Proceedings of the First International Conference on Product Design for Assembly, Newport, RI.

[10] Lee, K. and Gadh, R. (1996), "Computer Aided Design for Disassembly: A Destructive Approach", Proceedings of the IEEE International Symposium on Electronics and Environment, pp. 173-178.

[11] Nishiwaski, S. (1998), Optimum Structural Topology Design Considering Flexibility, University of Michigan Ph. D. dissertation.

[12] Nishiwaki; S:, Silva, E., Li, Y. and Kikuchi, N.(1998) "Topology optimization for flextensional actuators", Proceeding of AIAA symposium on Multi-disciplinary Optimization St. Louise 1998.

[13] Ishii, K., Eubanks, C., and DiMarco, P. (1994), "Design for Product Retirement and Material Life-cycle", Materials and Design. Vol. 15, pp. 225-233.

[14] Ishii, K. and Lee, B. (1996), "Reverse Fishbone Diagram: A Tool in Aid of Design for Product Retirement", Proceedings of the ASME Design Technical Conference, 96DETC/DFM-1272.

[15] Harjula, T., Rapoza, B., Knight, W. and Boothroyd, G. (1996), "Design for Disassembly and the Environment", Annals of the CIRP, Vol. 45, No. 1 pp. 109-114.

[16] Bensdoe, M.P. \& Kikuchi, N.(1988), "Generating Optimal Topologies in Structural Design Using a Homogenization Method", Computer Methods in Applied Mechanics and Engineering Vol. 71 pp. 197-224

[17] Suzuki, K. and Kikuchi, N. (1991), "A Homogenization Method for Shape and Topology Optimization", Computer Methods in Applied Mechanics and Engineering Vol. 93 pp. 291-318

[18] Huang, N. (1971), "On Principle of Stationary Mutual Complementary Energy and Its Application to Optimal Structural Design", Journal of Applied Mathematics and Physics, ZAMP 22 pp. 609-620

[19] Shield, R. T. and Prager, W. (1970), "Optimal Structural Design for Given Deflection", Journal of Applied Mathematics and Physics, ZAMP 21 pp. 513-523

[20] Li, Y., Chen, B. \& Kikuchi, N. (1999) "Topology Optimization of Mechanism with Thermal Actuation", Proceeding of the $4^{\text {th }}$ International conference on ECO materials. 